\title{
Homoclinic solutions for second-order Hamiltonian systems with periodic potential
}

Yiwei $Y e^{1 *}$

"Correspondence:
yeyiwei2011@126.com
'School of Mathematical Sciences,
Chongqing Normal University,
Chongqing, P.R. China

\begin{abstract}
In this paper, we study the second-order Hamiltonian systems

$$
\ddot{u}-L(t) u+\nabla W(t, u)=0, \quad t \in \mathbb{R},
$$

where $L \in C\left(\mathbb{R}, \mathbb{R}^{N \times N}\right)$ is a $T$-periodic and positive definite matrix for all $t \in \mathbb{R}$ and $W$ is superquadratic but does not satisfy the usual Ambrosetti-Rabinowitz condition at infinity. One ground homoclinic solution is obtained by applying the monotonicity trick of Jeanjean and the concentration-compactness principle. The main result improves the recent result of Liu-Guo-Zhang (Nonlinear Anal., Real World Appl. $36: 116-138,2017)$.
\end{abstract}

MSC: $37 \mathrm{~J} 45 ; 58 \mathrm{E} 05$

Keywords: Homoclinic solution; Ground state; Variational methods; Vanishing; Nonvanishing

\section{Introduction and main results}

Consider the second-order Hamiltonian systems

$$
\ddot{u}-L(t) u+\nabla W(t, u)=0,
$$

where $t \in \mathbb{R}, u \in \mathbb{R}^{N}, W \in C^{1}\left(\mathbb{R} \times \mathbb{R}^{N}, \mathbb{R}\right)$ and $\nabla W(t, x)$ denotes the gradient of $W$ with respect to $x$. Recall that a solution $u$ of system (1.1) is said to be homoclinic to 0 if $u \neq \equiv 0$ and $u(t) \rightarrow 0$ as $|t| \rightarrow \infty$. Furthermore, if $u$ minimizes the energy functional of (1.1) among all possible nontrivial homoclinic solutions, then $u$ is called a ground state homoclinic solution.

During the past two decades, there has been a remarkable amount of progress in the study of homoclinic motions of Hamiltonian systems, with many new ideas and methods being introduced; see, e.g., [2, 3, 5, 7, 11-13, 15, 17-21, 23] for second-order systems and $[4,6,14,16]$ for first-order systems. For $(1.1)$, most work considers the case where $L$ and $W$ depend periodically on $t$. Rabinowitz [12] prove the existence of one homoclinic solution of (1.1), which is obtained as the limit of $2 k T$ periodic solutions, under the following global Ambrosetti-Rabinowitz superquadratic condition:

(AR) $\exists \mu>2$ such that $0<\mu W(t, x) \leq(\nabla W(t, x), x)$ for all $(t, x) \in \mathbb{R} \times\left(\mathbb{R}^{N} \backslash\{0\}\right)$.

(c) The Author(s) 2018. This article is distributed under the terms of the Creative Commons Attribution 4.0 International License (http://creativecommons.org/licenses/by/4.0/), which permits unrestricted use, distribution, and reproduction in any medium, provided you give appropriate credit to the original author(s) and the source, provide a link to the Creative Commons license, and indicate if changes were made. 
The general Hamiltonian system $\ddot{u}+\nabla V(t, u)=f(t)$ has been studied in IzydorekJanczewska [7]. Besides some other conditions, they assume that $V \in C^{1}\left(\mathbb{R} \times \mathbb{R}^{N}, \mathbb{R}\right)$, $V(t, x)=-K(t, x)+W(t, x)$ is $T$-periodic in $t, K$ satisfies the pinching condition $b_{1}|x|^{2} \leq$ $K(t, x) \leq b_{2}|x|^{2}, W$ satisfies (AR) and $f \in L^{2}\left(\mathbb{R}, \mathbb{R}^{N}\right)$ small enough and prove the existence of one homoclinic orbit. Under (AR), Coti-Zelati and Rabinowitiz [2] establish the existence of infinitely many geometrically distinct homoclinic orbits by using a novel variational method in virtue of the famous "mountain pass" technique [1]. This result is deepened in Ding and Lee [5], where the authors find conditions weaker than (AR) and ensure the existence of infinitely many homoclinic orbits for both superquadratic and asymptotically quadratic cases. Recently, Wang [18] has studied problem (1.1) with periodicity. Instead of the common condition that $\lim _{|x| \rightarrow \infty} \frac{W(t, x)}{|x|^{2}}=+\infty$ uniformly in $t \in \mathbb{R}$, he uses a locally superquadratic condition that $\lim _{|x| \rightarrow \infty} \frac{W(t, x)}{|x|^{2}}=+\infty$ a.e. $t \in(a, b)$ for some $-\infty<a<b<+\infty$. See also $[3,13,15,17,19-21,23]$ for results concerning the nonperiodic case.

Our study is motivated by the recent result of Liu et al. [11], where the authors consider system (1.1) with the hypotheses that

$\left(L_{1}\right) L \in C\left(\mathbb{R}, \mathbb{R}^{N^{\times}}\right)$is $T$-periodic in $t$, and it is a symmetric and positive definite matrix for all $t \in \mathbb{R}$;

$\left(L_{2}\right)$ there exist constants $0<d_{1}<d_{2}$ such that

$$
d_{1}|x|^{2} \leq(L(t) x, x) \leq d_{2}|x|^{2}, \quad \forall(t, x) \in \mathbb{R} \times \mathbb{R}^{N}
$$

$\left(W_{1}\right) W \in C^{1}\left(\mathbb{R} \times \mathbb{R}^{N}, \mathbb{R}\right)$ and $W(t, x)$ is $T$-periodic in $t ;$

$\left(W_{2}\right) \quad \nabla W(t, x)=o(|x|)$ as $x \rightarrow 0$ uniformly in $t, W(t, 0)=0$ and $W(t, x) \geq 0$ for all $(t, x)$;

$\left(W_{3}\right) W(t, x) /|x|^{2} \rightarrow+\infty$ uniformly in $t$ as $|x| \rightarrow \infty$;

$\left(W_{4}\right) s \rightarrow s^{-1}(\nabla W(t, s x), x)$ is strictly increasing of $s>0$ for all $x \neq 0$ and $t \in \mathbb{R}$.

They prove the existence of one ground state homoclinic solution via the generalized $\mathrm{Ne}$ hari manifold developed by Szulkin and Weth.

In the present paper, we are interested in problem (1.1) with periodic potential and nonlinearities satisfying conditions which are more general than $\left(W_{3}\right)-\left(W_{4}\right)$. The same result is obtained by using a monotonicity trick due to Jeanjean [8] together with the concentration-compactness principle. Precisely, we make the following assumptions:

$\left(W_{5}\right) \lim _{|x| \rightarrow \infty} \frac{W(t, x)}{|x|^{2}}=+\infty$ for a.e. $t \in \mathbb{R}$

$\left(W_{6}\right)$ there exists $p>2$ such that $|\nabla W(t, x)| \leq C\left(1+|x|^{p-1}\right)$ for all $(t, x) \in \mathbb{R} \times \mathbb{R}^{N}$;

$\left(W_{7}\right)$ there exists $C_{0} \geq 1$ such that

$$
H(t, s x) \leq C_{0} H(t, x), \quad \forall(t, x) \in \mathbb{R} \times \mathbb{R}^{N}, s \in[0,1],
$$

where $H(t, x):=\frac{1}{2}(\nabla W(t, x), x)-W(t, x)$.

Theorem 1.1 Assume that $\left(L_{1}\right),\left(W_{1}\right)-\left(W_{2}\right)$ and $\left(W_{5}\right)-\left(W_{7}\right)$ are satisfied. Then system (1.1) has at least one ground state homoclinic solution.

\section{Remark 1.1}

(i) Condition $\left(W_{7}\right)$ is originally due to Jeanjean [8] for a semilinear problem setting on $\mathbb{R}^{N}$. Also, it is used in Liu and Li [10] for the $p$-Laplacian equation. In [22], infinitely 
many periodic solutions of (1.1) are obtained, provided that $W(t,-x)=W(t, x)$ and $\left(W_{3}\right)$ and $\left(W_{7}\right)$ are satisfied.

(ii) It turns out that if, for fixed $(t, x) \in \mathbb{R} \times \mathbb{R}^{N} \backslash\{0\}, s^{-1}(\nabla W(t, s x), x)$ is increasing for all $s>0$, then $\left(W_{7}\right)$ is satisfied. Hence Theorem 1.1 extends [11, Theorem 1.1].

Indeed, for $s \in(0,1]$, we obtain using $\left(W_{4}\right)$

$$
\begin{aligned}
H(t, x)-H(t, s x) \\
=\frac{1}{2}[(\nabla W(t, x), x)-(\nabla W(t, s x), s x)]-[W(t, x)-W(t, s x)] \\
=\left[\int_{0}^{1}(\nabla W(t, x), x) \tau d \tau-\int_{0}^{s} \frac{(\nabla W(t, s x), x)}{s} \tau d \tau-\int_{s}^{1} \frac{(\nabla W(t, \tau x), x)}{\tau} \tau d \tau\right] \\
=\int_{0}^{s}\left((\nabla W(t, x), x)-\frac{(\nabla W(t, s x), x)}{s}\right) \tau d \tau \\
\quad+\int_{s}^{1}\left((\nabla W(t, x), x)-\frac{(\nabla W(t, \tau x), x)}{\tau}\right) \tau d \tau \\
\geq 0 .
\end{aligned}
$$

If $s=0$, we see from the inequality

$$
\begin{aligned}
\frac{1}{2}(\nabla W(t, x), x) & =\int_{0}^{1}(\nabla W(t, x), x) \tau d \tau \\
& \geq \int_{0}^{1} \frac{(\nabla W(t, \tau x), x)}{\tau} \tau d \tau \\
& =W(t, x)-W(t, 0)
\end{aligned}
$$

that

$$
\frac{1}{2}(\nabla W(t, x), x)-W(t, x) \geq-W(t, 0), \quad \forall(t, x) \in \mathbb{R} \times \mathbb{R}^{N}
$$

i.e.,

$$
H(t, x) \geq H(t, 0), \quad \forall(t, x) \in \mathbb{R} \times \mathbb{R}^{N} .
$$

Therefore condition $\left(W_{7}\right)$ holds with $C_{0}=1$.

(iii) Comparing with the result of Liu et al. [11, Theorem 1.1], the advantage of our Theorem 1.1 is that the strictly increasing condition is removed. Thus our result applies to more general situations. We emphasize that this condition plays an essential role in the argument of Liu et al. [11]. Indeed, letting $S=\{u \in E:\|u\|=1\}$ and $\mathcal{N}=\left\{u \in E \backslash\{0\}:\left\langle I^{\prime}(u), u\right\rangle=0\right\}$, the starting point of their approach is to show that, for each $u \in S$, there exists an exactly one point $m(u) \in \mathcal{N}$. The uniqueness of $m(u)$ enables one to define a map $u \longmapsto m(u)$, which is important in the remaining proof. If $s \rightarrow s^{-1}(\nabla W(t, s x), x)$ is not strictly increasing, then $m(u)$ may not be unique and their argument collapses. 
Remark 1.2 There are functions $L$ and $W$ which match Theorem 1.1 but do not satisfy the results in $[2,3,5,7,11-13,15,17,19-21,23]$. For example, let $L(t)=I_{N}$, where $I_{N}$ denotes the identity matrix of order $N$, and

$$
W(t, x)=\left(1+\sin \frac{2 \pi}{T} t\right)|x|^{2} \ln \left(1+|x|^{2}\right), \quad \forall(t, x) \in \mathbb{R} \times \mathbb{R}^{N}
$$

Simple calculation shows that

$$
\begin{aligned}
& \nabla W(t, x)=\left(1+\sin \frac{2 \pi}{T} t\right)\left(2 x \ln \left(1+|x|^{2}\right)+\frac{2 x|x|^{2}}{1+|x|^{2}}\right), \\
& H(t, x)=\left(1+\sin \frac{2 \pi}{T} t\right) \frac{|x|^{4}}{1+|x|^{2}} .
\end{aligned}
$$

Then it is easy to check that $W$ satisfies $\left(W_{1}\right)-\left(W_{2}\right)$ and $\left(W_{5}\right)-\left(W_{7}\right)$. However, since $W\left(\frac{3}{4} T, x\right) \equiv 0$ for all $x \in \mathbb{R}^{N}$, it satisfies none of $(\mathrm{AR}),\left(W_{3}\right)$ and $\left(W_{4}\right)$.

Notations: " $\rightarrow$ " and " $\rightarrow$ " denote the strong convergence and the weak convergence, respectively. $C$ and $C_{i}(i=1,2, \ldots)$ denote various positive constants which may vary from line to line.

\section{Proof of Theorem 1.1}

We work in the Hilbert space

$$
E=\left\{u \in H^{1}\left(\mathbb{R}, \mathbb{R}^{N}\right): \int_{\mathbb{R}}\left[|\dot{u}|^{2}+(L(t) u, u)\right] d t<+\infty\right\}
$$

equipped with the inner product and norm

$$
\langle u, v\rangle=\int_{\mathbb{R}}[(\dot{u}, \dot{v})+(L(t) u, v)] d t, \quad\|u\|=\langle u, u\rangle^{1 / 2}
$$

for $u, v \in E$. By $\left(L_{1}\right), E$ is continuously embedded into $H^{1}\left(\mathbb{R}, \mathbb{R}^{N}\right)$, and hence $E$ is continuously embedded into $L^{s}\left(\mathbb{R}, \mathbb{R}^{N}\right)$ for $2 \leq s \leq \infty$, i.e., there exists $\tau_{s}>0$ such that

$$
\|u\|_{s} \leq \tau_{s}\|u\|, \quad \forall u \in E
$$

where $\|\cdot\|_{s}$ denotes the usual norm on $L^{s}\left(\mathbb{R}, \mathbb{R}^{N}\right)(2 \leq s \leq \infty)$.

Define the energy functional $\varphi: E \rightarrow \mathbb{R}$ by

$$
\begin{aligned}
\varphi(u) & =\frac{1}{2} \int_{\mathbb{R}}\left[|\dot{u}|^{2}+(L(t) u, u)\right] d t-\int_{\mathbb{R}} W(t, u) d t \\
& =\frac{1}{2}\|u\|^{2}-\int_{\mathbb{R}} W(t, u) d t .
\end{aligned}
$$

By $\left(W_{2}\right)$ and $\left(W_{6}\right)$, we find that, for any $\varepsilon>0$, there is $C_{\varepsilon}>0$ such that

$$
|\nabla W(t, x)| \leq \varepsilon|x|+C_{\varepsilon}|x|^{p-1}
$$


and

$$
|W(t, x)| \leq \varepsilon|x|^{2}+C_{\varepsilon}|x|^{p}
$$

for all $(t, x) \in \mathbb{R} \times \mathbb{R}^{N}$. Hence $\varphi \in C^{1}(E, \mathbb{R})$ and

$$
\left\langle\varphi^{\prime}(u), v\right\rangle=\langle u, v\rangle-\int_{\mathbb{R}}(\nabla W(t, u), v) d t \quad \forall u, v \in E
$$

It is routine to show that the nontrivial critical points of $\varphi$ on $E$ are homoclinic solutions of (1.1).

We shall prove that problem (1.1) has a mountain pass type solution. For this purpose, we apply the following theorem, which is given in [8].

Theorem 2.1 (see Jeanjean [8]) Let $(X,\|\cdot\|)$ be a Banach space and $J \subset \mathbb{R}^{+}$be an interval. Consider a family $\left(I_{\lambda}\right)_{\lambda \in J}$ of $C^{1}$ functionals on $X$ of the form

$$
I_{\lambda}(u)=A(u)-\lambda B(u), \quad \forall \lambda \in J
$$

where $B(u) \geq 0$ for all $u \in X, B(u) \rightarrow+\infty$ or $A(u) \rightarrow+\infty$ as $\|u\| \rightarrow \infty$. Assume that there are two points $v_{1}, v_{2} \in X$ such that

$$
c_{\lambda}:=\inf _{\gamma \in \Gamma} \max _{t \in[0,1]} I_{\lambda}(\gamma(t))>\max \left\{I_{\lambda}\left(v_{1}\right), I_{\lambda}\left(v_{2}\right)\right\}, \quad \forall \lambda \in J
$$

where $\Gamma=\left\{\gamma \in C([0,1], X): \gamma(0)=v_{1}, \gamma(1)=v_{2}\right\}$. Then, for almost every $\lambda \in J$, there is a sequence $\left\{v_{n}\right\} \subset X$ such that

(i) $\left(v_{n}\right)$ is bounded in $X$,

(ii) $I_{\lambda}\left(v_{n}\right) \stackrel{n}{\longrightarrow} c_{\lambda}$,

(iii) $I_{\lambda}^{\prime}\left(v_{n}\right) \stackrel{n}{\longrightarrow} 0$ in $X^{-1}$.

Moreover, the map $\lambda \rightarrow c_{\lambda}$ is continuous from the left.

For $\lambda \in[1,2]$, we define the family of functionals $\varphi_{\lambda}: E \rightarrow \mathbb{R}$ by

$$
\varphi_{\lambda}(u)=\frac{1}{2} \int_{\mathbb{R}}\left[|\dot{u}|^{2}+(L(t) u, u)\right] d t-\lambda \int_{\mathbb{R}} W(t, u) d t
$$

Lemma 2.1 Assume that $\left(L_{1}\right),\left(W_{2}\right),\left(W_{5}\right)$ and $\left(W_{6}\right)$ hold. Then

(i) There exists $u_{0} \in E \backslash\{0\}$ such that $\varphi_{\lambda}\left(u_{0}\right)<0$ for all $\lambda \in[1,2]$;

(ii) $c_{\lambda}:=\inf _{\gamma \in \Gamma} \max _{t \in[0,1]} \varphi_{\lambda}(\gamma(t))>\max \left\{\varphi_{\lambda}(0), \varphi_{\lambda}\left(u_{0}\right)\right\}$ for all $\lambda \in[1,2]$, where $\Gamma=\left\{\gamma \in C([0,1], E): \gamma(0)=0, \gamma(1)=u_{0}\right\}$.

Proof (i) Choose $e_{0} \in C_{0}^{\infty}(\mathbb{R}) \backslash\{0\}$. By $\left(W_{5}\right)$, Fatou's lemma and the fact $W \geq 0$, we have

$$
\lim _{s \rightarrow+\infty} \frac{\varphi_{\lambda}\left(s e_{0}\right)}{s^{2}} \leq \lim _{s \rightarrow+\infty} \frac{\varphi_{1}\left(s e_{0}\right)}{s^{2}} \leq \frac{1}{2}\left\|e_{0}\right\|^{2}-\lim _{s \rightarrow+\infty} \int_{e_{0} \neq 0} \frac{W\left(t, s e_{0}\right)}{\left(s e_{0}\right)^{2}} e_{0}^{2} d t<0
$$

for all $\lambda \in[1,2]$. So, we can take $s_{0}>0$ large enough such that $\varphi_{1}\left(s_{0} e_{0}\right)<0$. Then, setting $u_{0}=s_{0} e_{0}$, we see that $\varphi_{\lambda}\left(u_{0}\right) \leq \varphi_{1}\left(u_{0}\right)<0$ and (i) holds. 
(ii) Since, by (2.3) and (2.1),

$$
\left|\int_{\mathbb{R}} W(t, u) d t\right| \leq \varepsilon\|u\|_{2}^{2}+C_{\varepsilon}\|u\|_{p}^{p}=o\left(\|u\|^{2}\right) \text { as } u \rightarrow 0,
$$

we deduce that there exist constants $\alpha, \rho>0$ such that $\left.\varphi_{\lambda}\right|_{\|u\|=\rho} \geq \alpha$ for all $\lambda \in[1,2]$. Hence, letting $\Gamma=\left\{\gamma \in C([0,1], E): \gamma(0)=0, \gamma(1)=u_{0}\right\}$, we obtain

$$
c_{\lambda}=\inf _{\gamma \in \Gamma} \max _{t \in[0,1]} \varphi_{\lambda}(\gamma(t))>\max \left\{\varphi_{\lambda}(0), \varphi_{\lambda}\left(u_{0}\right)\right\}, \quad \forall \lambda \in[1,2]
$$

and the proof is complete.

Combining Lemma 2.1 and Theorem 2.1, we have the following.

Lemma 2.2 Assume that $\left(L_{1}\right),\left(W_{2}\right),\left(W_{5}\right)$ and $\left(W_{6}\right)$ are satisfied. Then, for a.e. $\lambda \in[1,2]$, there is a bounded sequence $\left(u_{n}\right) \subset E$ such that $\varphi_{\lambda}\left(u_{n}\right) \rightarrow c_{\lambda}$ and $\varphi^{\prime}\left(u_{n}\right) \rightarrow 0$ as $n \rightarrow \infty$.

Let $\left(u_{n}\right)$ be a bounded sequence in $E$, we say that $\left(u_{n}\right)$ is vanishing if, for each $R>0$, $\lim _{n \rightarrow \infty} \sup _{y \in \mathbb{R}} \int_{y-R}^{y+R}\left|u_{n}\right|^{2} d t=0$; and $\left(u_{n}\right)$ is nonvanishing if there exist $\sigma>0, R>0$ and $\left(y_{n}\right) \subset \mathbb{R}$ such that $\liminf _{n \rightarrow \infty} \int_{y_{n}-R}^{y_{n}+R}\left|u_{n}\right|^{2} d t \geq \sigma$. In the vanishing case, we have the following result, which is a special case of Lions [9].

Lemma 2.3 (see [9]) Let $\left(u_{n}\right) \subset E$ be a bounded sequence, if

$$
\lim _{n \rightarrow \infty} \sup _{y \in \mathbb{R}} \int_{y-R}^{y+R}\left|u_{n}\right|^{2} d t=0
$$

then $u_{n} \rightarrow 0$ in $L^{s}\left(\mathbb{R}, \mathbb{R}^{N}\right)$ for $2<s<\infty$.

Lemma 2.4 Assume that $\left(L_{1}\right),\left(W_{2}\right)$ and $\left(W_{6}\right)$ hold. Then, for any bounded vanishing sequence $\left(u_{n}\right) \subset E$, we have

$$
\lim _{n \rightarrow \infty} \int_{\mathbb{R}} H\left(t, u_{n}\right) d t=0
$$

Proof It follows from (2.2) and (2.3) that

$$
\left|\int_{\mathbb{R}}\left(\nabla W\left(t, u_{n}\right), u_{n}\right) d t\right| \leq \varepsilon\left\|u_{n}\right\|_{2}^{2}+C_{\varepsilon}\left\|u_{n}\right\|_{p}^{p}
$$

and

$$
\left|\int_{\mathbb{R}} W\left(t, u_{n}\right) d t\right| \leq \varepsilon\left\|u_{n}\right\|_{2}^{2}+C_{\varepsilon}\left\|u_{n}\right\|_{p}^{p}
$$

Since $\left(u_{n}\right)$ is vanishing, by Lemma 2.3 , we deduce that

$$
\int_{\mathbb{R}}\left(\nabla W\left(t, u_{n}\right), u_{n}\right) d t \rightarrow 0 \quad \text { and } \int_{\mathbb{R}} W\left(t, u_{n}\right) d t \rightarrow 0
$$

as $n \rightarrow \infty$, and the conclusion follows. 
Lemma 2.5 Assume that $\left(L_{1}\right),\left(W_{1}\right)-\left(W_{2}\right)$ and $\left(W_{6}\right)-\left(W_{7}\right)$ hold. Then, for all bounded sequence $\left(u_{n}\right) \subset$ E satisfying

$$
0<\lim _{n \rightarrow \infty} \varphi_{\lambda}\left(u_{n}\right) \leq c_{\lambda} \quad \text { and } \quad \lim _{n \rightarrow \infty} \varphi_{\lambda}^{\prime}\left(u_{n}\right)=0
$$

there exists $\left(y_{n}\right) \subset \mathbb{Z}$ such that, up to a subsequence, $\tilde{u}_{n}(t):=u_{n}\left(t+y_{n} T\right)$ satisfies

$$
\tilde{u}_{n} \rightarrow u_{\lambda} \neq 0, \quad \varphi_{\lambda}\left(u_{\lambda}\right) \leq c_{\lambda} \text { and } \varphi_{\lambda}^{\prime}\left(u_{\lambda}\right)=0 .
$$

Proof Since $\left\langle\varphi_{\lambda}^{\prime}\left(u_{n}\right), u_{n}\right\rangle \rightarrow 0$, we have

$$
\lim _{n \rightarrow \infty} \lambda \int_{\mathbb{R}}\left[\frac{1}{2}\left(\nabla W\left(t, u_{n}\right), u_{n}\right)-W\left(t, u_{n}\right)\right] d t=\lim _{n \rightarrow \infty} \varphi_{\lambda}\left(u_{n}\right)>0
$$

Combining this and Lemma 2.4, we see that $\left(u_{n}\right)$ is nonvanishing. Hence, there exist $\sigma>0$, $R>0$ and $\left(\bar{y}_{n}\right) \subset \mathbb{R}$ such that

$$
\liminf _{n \rightarrow \infty} \int_{\bar{y}_{n}-R}^{\bar{y}_{n}+R}\left|u_{n}\right|^{2} d t \geq \sigma>0
$$

We may choose $\left(y_{n}\right) \subset \mathbb{Z}$ such that, letting $\tilde{u}_{n}(t):=u_{n}\left(t+y_{n} T\right)$,

$$
\liminf _{n \rightarrow \infty} \int_{-2 R}^{2 R}\left|\tilde{u}_{n}\right|^{2} d t \geq \frac{\sigma}{2}>0
$$

Noticing $L$ and $W$ is $T$-periodic in $t$, we have $\left\|\tilde{u}_{n}\right\|=\left\|u_{n}\right\|, \varphi_{\lambda}\left(\tilde{u}_{n}\right)=\varphi_{\lambda}\left(u_{n}\right)$ and

$$
\varphi_{\lambda}^{\prime}\left(\tilde{u}_{n}\right) \rightarrow 0 \quad \text { as } n \rightarrow \infty
$$

Indeed, for each $\psi \in E$, take $\psi_{n}(t):=\psi\left(t-y_{n}\right)$. It is easy to check that $\left\|\psi_{n}\right\|=\|\psi\|$ and

$$
\begin{aligned}
\left|\left\langle\varphi_{\lambda}^{\prime}\left(\tilde{u}_{n}\right), \psi\right\rangle\right| & =\int_{\mathbb{R}}\left[\left(\dot{\tilde{u}}_{n}, \dot{\psi}\right)+\left(L(t) \tilde{u}_{n}, \psi\right)-\lambda\left(\nabla W\left(t, \tilde{u}_{n}\right), \psi\right)\right] d t \\
& =\int_{\mathbb{R}}\left[\left(\dot{u}_{n}, \dot{\psi}_{n}\right)+\left(L(t) u_{n}, \psi_{n}\right)-\lambda\left(\nabla W\left(t, u_{n}\right), \psi_{n}\right)\right] d t \\
& \left.=\| \varphi_{\lambda}^{\prime}\left(u_{n}\right), \psi_{n}\right\rangle \mid \\
& \leq\left\|\varphi_{\lambda}^{\prime}\left(u_{n}\right)\right\|\left\|\psi_{n}\right\| \\
& =\left\|\varphi_{\lambda}^{\prime}\left(u_{n}\right)\right\|\|\psi\| \rightarrow 0,
\end{aligned}
$$

which gives (2.5). Since $\left(\tilde{u}_{n}\right)$ is still bounded, going if necessary to a subsequence, we may assume that there is $u_{\lambda} \in E$ such that

$$
\begin{array}{ll}
\tilde{u}_{n} \rightarrow u_{\lambda} & \text { in } E, \\
\tilde{u}_{n} \rightarrow u_{\lambda} & \text { in } L_{\text {loc }}^{s}\left(\mathbb{R}, \mathbb{R}^{N}\right) \text { for } s \in(1, \infty), \\
\tilde{u}_{n} \rightarrow u_{\lambda} & \text { a.e. in } \mathbb{R},
\end{array}
$$


and $u_{\lambda} \neq 0$ by (2.4). It follows from (2.2) and (2.6) that

$$
\left|\int_{\mathbb{R}}\left(\nabla W\left(t, \tilde{u}_{n}\right)-\nabla W\left(t, u_{\lambda}\right), \psi\right) d t\right| \rightarrow 0, \quad \forall \psi \in C_{0}^{\infty}(\mathbb{R})
$$

which implies that $\varphi_{\lambda}^{\prime}$ is weakly sequentially continuous. Thus, by (2.5),

$$
\varphi_{\lambda}^{\prime}\left(u_{\lambda}\right)=0
$$

Finally, by $\left(W_{7}\right)$ and Fatou's lemma,

$$
\begin{aligned}
c_{\lambda} & \geq \lim _{n \rightarrow \infty}\left(\varphi_{\lambda}\left(\tilde{u}_{n}\right)-\frac{1}{2}\left\langle\varphi_{\lambda}^{\prime}\left(\tilde{u}_{n}\right), \tilde{u}_{n}\right\rangle\right) \\
& =\lim _{n \rightarrow \infty} \lambda \int_{\mathbb{R}} H\left(t, \tilde{u}_{n}\right) d t \\
& \geq \lambda \int_{\mathbb{R}} H\left(t, u_{\lambda}\right) d t \\
& =\varphi_{\lambda}\left(u_{\lambda}\right)-\frac{1}{2}\left\langle\varphi_{\lambda}^{\prime}\left(u_{\lambda}\right), u_{\lambda}\right\rangle \\
& =\varphi_{\lambda}\left(u_{\lambda}\right) .
\end{aligned}
$$

The proof is complete.

Remark 2.1 If the sequence $\left(u_{n}\right)$ in Lemma 2.5 is nonvanishing, then the assumption $0<$ $\lim _{n \rightarrow \infty} \varphi_{\lambda}\left(u_{n}\right)$ can be omitted.

As a consequence of Lemmas 2.2 and 2.5, we have the following.

Lemma 2.6 Assume that $\left(L_{1}\right),\left(W_{1}\right)-\left(W_{2}\right)$ and $\left(W_{6}\right)-\left(W_{7}\right)$ hold. Then there exist $\left(\lambda_{n}\right) \subset$ $[1,2]$ and $\left(u_{n}\right) \subset E \backslash\{0\}$ such that

$$
\lambda_{n} \rightarrow 1, \quad \varphi_{\lambda_{n}}\left(u_{n}\right) \leq c_{\lambda_{n}} \quad \text { and } \quad \varphi_{\lambda_{n}}^{\prime}\left(u_{n}\right)=0 .
$$

Lemma 2.7 The sequence $\left(u_{n}\right)$ obtained in Lemma 2.6 is bounded.

Proof Suppose by contradiction that $\left\|u_{n}\right\| \rightarrow \infty(n \rightarrow \infty)$. Set $w_{n}=u_{n} /\left\|u_{n}\right\|$. Then $\left\|w_{n}\right\|=$ 1 , and by a Lions' concentration-compactness principle [9], either $\left(w_{n}\right)$ is vanishing or it is nonvanishing. Hence the proof of the lemma will be completed if we show that $\left(w_{n}\right)$ is neither vanishing nor nonvanishing.

Assume $\left(w_{n}\right)$ is vanishing. As in $[8,24]$, we choose a sequence $s_{n} \in[0,1]$ such that

$$
\varphi_{\lambda_{n}}\left(s_{n} u_{n}\right)=\max _{t \in[0,1]} \varphi_{\lambda_{n}}\left(s u_{n}\right)
$$

For any $M>0$, let $v_{n}=\left(2 \sqrt{M} /\left\|u_{n}\right\|\right) u_{n}=2 \sqrt{M} w_{n}$. Since $\left(v_{n}\right)$ is vanishing and bounded, by Lemma 2.3 and (2.3), we have

$$
\int_{\mathbb{R}} W\left(t, v_{n}\right) d t \rightarrow 0 \quad \text { as } n \rightarrow \infty
$$


Now, for large $n, \frac{2 \sqrt{M}}{\left\|u_{n}\right\|} \in(0,1)$, and by the definition of $s_{n}$, we deduce that

$$
\varphi_{\lambda_{n}}\left(s_{n} u_{n}\right) \geq \varphi_{\lambda_{n}}\left(v_{n}\right)=2 M-\lambda_{n} \int_{\mathbb{R}} W\left(t, v_{n}\right) d t \geq M
$$

which implies that

$$
\varphi_{\lambda_{n}}\left(s_{n} u_{n}\right) \rightarrow+\infty \quad \text { as } n \rightarrow \infty
$$

Observing $\varphi_{\lambda_{n}}(0)=0$ and $\varphi_{\lambda_{n}}\left(u_{n}\right) \leq c_{\lambda_{n}}$, we get $s_{n} \in(0,1)$ and

$$
\left\langle\varphi_{\lambda_{n}}^{\prime}\left(s_{n} u_{n}\right), s_{n} u_{n}\right\rangle=\left.s_{n} \frac{d}{d s}\right|_{s=s_{n}} \varphi_{\lambda_{n}}\left(s u_{n}\right)=0
$$

Therefore, using (2.11) and (2.12), we deduce

$$
\begin{aligned}
\int_{\mathbb{R}} H\left(t, s_{n} u_{n}\right) d t & =\frac{1}{\lambda_{n}}\left[\varphi_{\lambda_{n}}\left(s_{n} u_{n}\right)-\frac{1}{2}\left\langle\varphi_{\lambda_{n}}^{\prime}\left(s_{n} u_{n}\right), s_{n} u_{n}\right\rangle\right] \\
& =\frac{1}{\lambda_{n}} \varphi_{\lambda_{n}}\left(s_{n} u_{n}\right) \\
& \rightarrow+\infty \quad \text { as } n \rightarrow \infty .
\end{aligned}
$$

However, it follows from $(2.9)$ and $\left(W_{7}\right)$ that

$$
\int_{\mathbb{R}} H\left(t, s_{n} u_{n}\right) d t \leq C_{0} \int_{\mathbb{R}} H\left(t, u_{n}\right) d t \leq \frac{C_{0}}{\lambda_{n}}\left[\varphi_{\lambda_{n}}\left(u_{n}\right)-\frac{1}{2}\left\langle\varphi_{\lambda_{n}}^{\prime}\left(u_{n}\right), u_{n}\right\rangle\right] \leq C, \quad \forall n \in \mathbb{N},
$$

yielding a contradiction.

Assume $\left(w_{n}\right)$ is nonvanishing. Then, as in the proof of (2.6), by the translation invariance of problem (1.1), one has $w_{n} \rightarrow w$ in $E$ and $w_{n}(t) \rightarrow w(t)$ a.e. in $\mathbb{R}$ for some $w \in E \backslash\{0\}$. On the set $\{t \in \mathbb{R}: w(t) \neq 0\}$, one has $\left|u_{n}(t)\right| \rightarrow \infty$, and then, by $\left(W_{5}\right)$,

$$
\frac{W\left(t, u_{n}\right)}{\left|u_{n}\right|^{2}}\left|w_{n}\right|^{2} \rightarrow+\infty \quad \text { as } n \rightarrow \infty
$$

Therefore, taking into account $|\Omega|>0$ and using Fatou's lemma, we obtain

$$
\int_{\mathbb{R}} \frac{W\left(t, u_{n}\right)}{\left\|u_{n}\right\|^{2}} d t \geq \int_{\Omega} \frac{W\left(t, u_{n}\right)}{\left|u_{n}\right|^{2}}\left|w_{n}\right|^{2} d t \rightarrow+\infty \quad \text { as } n \rightarrow \infty .
$$

On the other hand, since $\varphi_{\lambda_{n}}\left(u_{n}\right) \rightarrow c_{\lambda_{n}}$, we deduce that

$$
\lim _{n \rightarrow \infty} \int_{\mathbb{R}} \frac{W\left(t, u_{n}\right)}{\left\|u_{n}\right\|^{2}} d t=\frac{1}{2}
$$

a contradiction.

Proof of Theorem 1.1 First we show that $\varphi$ has a nontrivial critical point. By Lemma 2.6, we have, for any $v \in E$,

$$
\left\langle\varphi^{\prime}\left(u_{n}\right), v\right\rangle=\left\langle\varphi_{\lambda_{n}}^{\prime}\left(u_{n}\right), v\right\rangle+\left(\lambda_{n}-1\right) \int_{\mathbb{R}}\left(\nabla W\left(t, u_{n}\right), v\right) d t \rightarrow 0 \quad \text { as } n \rightarrow \infty
$$


Hence $\varphi^{\prime}\left(u_{n}\right) \rightarrow 0$. Since $\left\langle\varphi_{\lambda_{n}}^{\prime}\left(u_{n}\right), u_{n}\right\rangle=0$, it follows from (2.2) that

$$
\left\|u_{n}\right\|^{2}=\lambda_{n} \int_{\mathbb{R}}\left(\nabla W\left(t, u_{n}\right), u_{n}\right) d t \leq \varepsilon \tau_{2}^{2}\left\|u_{n}\right\|^{2}+C_{\varepsilon} \tau_{p}^{p}\left\|u_{n}\right\|^{p}
$$

which implies that $\left\|u_{n}\right\| \geq C_{1}(\forall n \in \mathbb{N})$ for some $C_{1}>0$. If $\left(u_{n}\right)$ is vanishing, by Lemma 2.3, the middle term of (2.14) tends to 0 , and then $u_{n} \rightarrow 0$, a contradiction. Thus $\left(u_{n}\right)$ is nonvanishing. Proceeding as in the proof of Lemma 2.5 , we conclude that there exist $\left(y_{n}\right) \subset \mathbb{Z}$ such that if $\tilde{u}_{n}(t)=u_{n}\left(t+y_{n} T\right)$, then $\tilde{u}_{n} \rightarrow \tilde{u} \neq 0$ and $\varphi^{\prime}(\tilde{u})=0$.

Next we prove the existence of ground homoclinic solution of (1.1). Let

$$
\mathcal{N}:=\left\{u \in E \backslash\{0\}: \varphi^{\prime}(u)=0\right\}
$$

and $m=\inf _{u \in \mathcal{N}} \varphi(u)$. Using $\left(W_{7}\right)$ and the fact $\tilde{u} \in \mathcal{N}$, we get $0 \leq m \leq \varphi(\tilde{u})$. By the definition of $m$, there is a sequence $\left(v_{n}\right) \subset \mathcal{N}$ such that $\varphi\left(v_{n}\right) \rightarrow m$ as $n \rightarrow \infty$. Following the same procedures as the proof of Lemma 2.7, we have $\left(v_{n}\right)$ is bounded. Since $\left(v_{n}\right) \subset \mathcal{N}, \varphi^{\prime}\left(v_{n}\right)=0$, similar to (2.14), we deduce that $\left\|v_{n}\right\| \geq C_{2}>0$ for all $n$ and $\left(v_{n}\right)$ is nonvanishing. Hence, arguing as in (2.6)-(2.8), we find that there is $\tilde{v} \in E \backslash\{0\}$ such that $\varphi^{\prime}(\tilde{v})=0$ and $\varphi(\tilde{v}) \leq m$. Noting $\tilde{v} \in \mathcal{N}$, one has $\varphi(\tilde{v}) \geq m$. Thus $\varphi(\tilde{v})=m$. This completes the proof.

\section{Acknowledgements}

The author would like to thank the handling editors and the anonymous reviewers.

\section{Funding}

This work is partially supported by the National Natural Science Foundation of China (No. 11601049), Chongqing Natural Science Foundation and Frontier Research Planning Project (No. cstc2015jcyjA00014), and the Scientific and

Technological Research Program of Chongqing Municipal Education Commission (No. KJ1500313).

\section{Availability of data and materials}

Not applicable.

\section{Competing interests}

The author declares that there are no competing interests.

\section{Authors' contributions}

The author conceived of the study, drafted the manuscript, read and approved the final manuscript.

\section{Publisher's Note}

Springer Nature remains neutral with regard to jurisdictional claims in published maps and institutional affiliations.

Received: 7 October 2018 Accepted: 3 December 2018 Published online: 11 December 2018

\section{References}

1. Ambrosetti, A., Rabinowitz, P.: Dual variational methods in critical point theory and applications. J. Funct. Anal. 14 349-381 (1973)

2. Coti-Zelati, V., Rabinowitz, P.: Homoclinic orbits for second order Hamiltonian systems possessing superquadratic potentials. J. Am. Math. Soc. 4, 693-727 (1991)

3. Ding, Y:: Existence and multiplicity results for homoclinic solutions to a class of Hamiltonian systems. Nonlinear Anal. 25, 1095-1113 (1995)

4. Ding, Y., Jeanjean, L.: Homoclinic orbits for a nonperiodic Hamiltonian system. J. Differ. Equ. 237, 473-490 (2007)

5. Ding, Y., Lee, C.: Homoclinics for asymptotically quadratic and superquadratic Hamiltonian systems. Nonlinear Anal. 71, 1395-1413 (2009)

6. Hofer, H., Wysocki, K.: First order elliptic systems and the existence of homoclinic orbits in Hamiltonian systems. Math. Ann. 288(1), 483-503 (1990). https://doi.org/10.1007/BF01444543

7. Izydorek, M., Janczewska, J.: Homoclinic solutions for a class of the second order Hamiltonian systems. J. Differ. Equ. 219, 375-389 (2005)

8. Jeanjean, L:: On the existence of bounded Palais-Smale sequences and application to a Landesman-Lazer-type problem set on $R^{N}$. Proc. R. Soc. Edinb. A 129, 787-809 (1999). https://doi.org/10.1017/S0308210500013147

9. Lions, P.L.: The concentration-compactness principle in the calculus of variations. The locally compact cases, Part II. Ann. Inst. Henri Poincaré, Anal. Non Linéaire 1(4), 223-283 (1984) 
10. Liu, S., Li, S.: Infinitely many solutions for a superlinear elliptic equation. Acta Math. Sinica (Chin. Ser.) 46(4), 625-630 (2003)

11. Liu, Z., Guo, S., Zhang, Z.: Homoclinic orbits for the second-order Hamiltonian systems. Nonlinear Anal., Real World Appl. 36, 116-138 (2017)

12. Rabinowitz, P.H.: Homoclinic orbits for a class of Hamiltonian systems. Proc. R. Soc. Edinb. A 114, 33-38 (1990)

13. Rabinowitz, P.H., Tanaka, K.: Some results on connecting orbits for a class of Hamiltonian systems. Math. Z. 206, 473-499 (1991)

14. Séré, E.: Existence of infinitely many homoclinic orbits in Hamiltonian systems. Math. Z. 209, 27-42 (1992)

15. Sun, J., Wu, T.: Multiplicity and concentration of homoclinic solutions for some second order Hamiltonian systems. Nonlinear Anal. 114, 105-115 (2015)

16. Szulkin, A., Zou, W.: Homoclinic orbits for asymptotically linear Hamiltonian systems. J. Funct. Anal. 187, 25-41 (2001)

17. Tang, X.: Infinitely many homoclinic solutions for a second-order Hamiltonian system. Math. Nachr. 289, 116-127 (2016)

18. Wang, X.: Local super-quadratic conditions on homoclinic solutions for a second-order Hamiltonian system. Appl. Math. Lett. 75, 7-12 (2018)

19. Wu, D.-L., Li, C., Yuan, P.: Multiplicity solutions for a class of fractional Hamiltonian systems with concave-convex potentials. Mediterr. J. Math. 15, Article ID 35 (2018). https://doi.org/10.1007/s00009-018-1079-y

20. Wu, D.-L., Tang, C.-L., Wu, X.-P.: Homoclinic orbits for a class of second-order Hamiltonian systems with concave-convex nonlinearities. Electron. J. Qual. Theory Differ. Equ. 2018, Article ID 6 (2018). https://doi.org/10.14232/ejqtde.2018.1.6

21. Ye, Y., Tang, C.-L.: New existence and multiplicity results of homoclinic orbits for a class of second order Hamiltonian systems. Chaos Solitons Fractals 69, 151-159 (2014)

22. Ye, Y., Tang, C.-L.: Infinitely many periodic solutions of non-autonomous second-order Hamiltonian systems. Proc. R. Soc. Edinb. A 144, 205-223 (2014)

23. Ye, Y., Tang, C.-L.: Multiple homoclinic solutions for second-order perturbed Hamiltonian systems. Stud. Appl. Math. $132,112-137(2014)$

24. Zou, W.: Variant fountain theorems and their applications. Manuscr. Math. 104, 343-358 (2001)

\section{Submit your manuscript to a SpringerOpen ${ }^{\circ}$ journal and benefit from:}

- Convenient online submission

- Rigorous peer review

- Open access: articles freely available online

- High visibility within the field

- Retaining the copyright to your article

Submit your next manuscript at $\boldsymbol{\nabla}$ springeropen.com 